HELMINTHOLOGIA, 53, 3: 211 - 223, 2016

\title{
Review
}

\section{Checklist of sea turtles endohelminth in Neotropical region}

\author{
M. R. WERNECK' ${ }^{1 *}$ R. J. DA SILVA²
}

'BW Veterinary Consulting. Rua Ponciano Eugênio Duarte 203, Centro, Zip Code: 11680-000, Ubatuba, São Paulo State, Brazil, *E-mail: max@bwvet.com.br, ${ }^{2}$ São Paulo State University (UNESP), Institute of Biosciences, Department of Parasitology, Laboratory of Wild Animals Parasitology, Botucatu, São Paulo State, Brazil

\section{Article info}

Received December 17, 2015 Accepted May 6, 2016

\begin{abstract}
Summary
This paper presents a list of parasites described in sea turtles from the Neotropical region. Through the review of literature the occurrence of 79 taxa of helminthes parasites were observed, mostly consisting of the Phylum Platyhelminthes with 76 species distributed in 14 families and 2 families of the Phylum Nematoda within 3 species. Regarding the parasite records, the most studied host was the green turtle (Chelonia mydas) followed by the hawksbill turtle (Eretmochelys imbricata), olive ridley turtle (Lepidochelys olivacea), loggerhead turtle (Caretta caretta) and leatherback turtle (Dermochelys coriacea). Overall helminths were reported in 12 countries and in the Caribbean Sea region. This checklist is the largest compilation of data on helminths found in sea turtles in the Neotropical region. Keywords: checklist; helminths; marine turtles; parasites
\end{abstract}

\section{Introduction}

The Neotropical region corresponds to Central and South America, where five species of sea turtles are found: Green turtle (CheIonia mydas Linnaeus, 1758), loggerhead turtle (Caretta caretta Linnaeus, 1758), hawksbill turtle (Eretmochelys imbricata Linnaeus, 1766), Olive ridley turtle (Lepidochelys olivacea Eschscholtz, 1829) and leatherback turtle (Dermochelys coriacea Vandelli, 1761) (Lutz \& Musick, 1996). The International Union for Conservation of Nature (IUCN, 2015) considers C. caretta, C. mydas and $L$. olivacea as endangered and both $D$. coriacea and $E$. imbricata as critically threatened.

Parasitological analyses of sea turtles have been conducted for many years and the results have contributed to a better understanding of the helminths of this group of hosts. Thus, the aim of this paper was to offer a comprehensive bibliographic review of the species of endohelminths found in sea turtles in the Neotropics.

\section{Material and Methods}

This checklist was developed using only scientific papers on endohelminths identified in sea turtles published between 1911 and 2016. Dissertations, theses and abstracts from conferences were not considered.

The data are presented in two forms: The first part of the paper presents a list of helminths (and synonyms) separated by family, genus and species and includes the infection site, location, infected host and author of the reference. The second part presents the hosts and a list of helminths reported in these hosts. For the classification, the taxonomic proposal presented by the World Register of Marine Species (WoRMS, 2015) was used.

\section{Results}

This checklist records the occurrence of 79 taxa of parasites. Se 
venty six represent the phylum Platyhelminthes (one from the order Aspidogastrida, 11 from the order Diplostomida and 64 from the order Plagiorchiida) represented by 14 families. Three represent the phylum Nematoda, in which two families have been found in the sea turtles C. mydas, C. caretta, E. imbricata, L. olivacea and $D$. coriacea. Overall helminths are reported in 12 countries and three are reported for the region in which the present study took place (i.e. the Caribbean Sea).

\section{Parasite-Host list}

Phylum Platyhelminthes Gegenbaur, 1859

Class Trematoda Rudolphi, 1808

Subclass Aspidogastrea Faust \& Tang, 1936

Order Aspidogastrida Skrjabin \& Guschanskaja, 1962

Superfamily Apidogastroidea Poche, 1907

Family Aspidogastridae Poche, 1907

Lophotaspis vallei (Stossich, 1899)

Site of infection: Esophagus and stomach.

Host and distribution: C. caretta from Brazil (Araújo, 1941)

Subclass Digenea Carus, 1863

Order Plagiorchiida La Rue, 1957

Suborder Echinostomata Szidat, 1939

Super Family Echinostomatoidea Looss, 1902

Family Calycodidae Dollfus. 1929

Calycodes anthos (Braun, 1899) Looss, 1901.

Site of infection: Small and large intestine.

Host and distribution: C. caretta from Brazil (Werneck et al.. 2008a), C. mydas from Panamá (Caballero et al.,1955) and Brazil (Binoti et al., 2016); D. coriacea from Uruguay (Werneck et al., 2012) and L. olivacea from México (Pérez-Ponce de Leon et al., 1996).

Calycodes caborojoensis Fischthal e Acholonu, 1976

Site of infection: small intestine of $E$ imbricata from Puerto Rico

(Fischthal \& Acholonu, 1976; Dyer et al.,1995a).

Familia Rhytidodidae Odhner, 1926

Rhytidodes gelatinosus (Rudolphi, 1819) Looss, 1901

Site of infection: Stomach and intestine.

Host and distribution: C. caretta from Brazil (Viana, 1924; Travassos et al., 1969); C. mydas from Panamá (Caballero, 1954) and Brazil (Werneck \& Silva, 2015) and E. imbricata from Puerto Rico (Fischthal \& Acholonu, 1976; Dyer et al., 1995a) and Cuba (Vigueras, 1955).

Rhytidodoides intestinalis Price, 1939

Site of infection: Gall bladder.

Host and distribution: C. mydas from Panamá (Caballero, 1954) and Costa Rica (Santoro et al., 2006).
Rhytidodoides similis Price, 1939

Site of infection: Gall bladder.

Host and distribution: C. mydas from Panamá (Caballero, 1954), Costa Rica (Santoro et al., 2006) and Brazil (Werneck et al., 2015a).

\section{Suborder Hemiurata Markevitsch 1951}

Superfamily Hemiuroidea Looss, 1899

Family Sclerodistomidae Odner, 1927

Prosorchis psenopsis Yamaguti, 1934

Site of infection: Stomach.

Host and distribution: L. olivacea from México (Pérez-Ponce de Leon et al., 1996).

Suborder Pronocephalata Olson, Cribb, Tkach, Bray \& Littlewood, 2003

Superfamily Paramphistomoidea Fischoeder, 1901

Family Cladorchiidae Fischoeder, 1901

Schizamphistomum erratum Blair, 1983

(Synonym: Schizamphistomoides erratum Blair, 1983)

Site of infection: Intestine.

Host and distribution: C. mydas from Costa Rica (Santoro et al., 2006).

Schizamphistomoides spinulosum (Looss, 1901) Stunkard, 1925 Site of infection: Large intestine.

Host and distribution: C. mydas from Panamá (Caballero et al., 1955).

Schizamphistomum scleroporum (Creplin, 1844) Looss, 1912

(Synonym: Schizamphistomoides chelonei Gupta 1961)

Site of infection: Stomach and intestine.

Host and distribution: C. mydas from Brazil (Werneck \& Silva 2015), Costa Rica (Santoro et al., 2006), Cuba (Groschaft et al.,1977) and Trinidade (The West Indies) (Gupta, 1961) and E. imbricata from Puerto Rico (Fischthal \& Acholonu, 1976).

Schizamphistomum sp. Looss, 1912

Site of infection: Intestine.

Host and distribution: C. mydas from Brazil (Binoti et al., 2016) and Puerto Rico (Dyer et al., 1995b).

Family Microscaphidiidae Looss, 1900

Angiodictyum anteroporum Chattopadhyaya, 1972 (Taxon inquirendum)

Site of infection: Large intestine.

Host and distribution: E. imbricata from Puerto Rico (Dyer et al., 1995a).

Angiodictyum longum Blair, 1986

Site of infection: Small intestine.

Host and distribution: C. mydas from Brazil (Werneck \& Silva 2015; Binoti et al., 2016). 
Angiodictyum mooreae Dyer, Williams \& Bunkley-Williams, 1995 Site of infection: Large intestine.

Host and distribution: E. imbricata from Puerto Rico (Dyer et al., 1995c).

Angiodictyum parallelum (Looss, 1901) Looss, 1902

(Synonym: Microscaphidium parallelum Looss, 1901)

Site of infection: Small and large intestine.

Host and distribution:, C. mydas from Brazil (Werneck \& Silva, 2015) and Puerto Rico (Dyer et al., 1991) and E. imbricata from Puerto Rico (Dyer et al., 1995c).

Deuterobaris chelonei Gupta, 1961.

Site of infection: Intestine.

Host and distribution: C. mydas from Trinidad (The West India) (Gupta, 1961).

Deuterobaris intestinalis Mehrotra, 1973

Site of infection: Intestine.

Host and distribution: C. mydas from Costa Rica (Santoro et al., 2006).

Deuterobaris proteus (Brandes, 1891) Looss, 1900

Site of infection: Esophagus, stomach, small and large intestine.

Host and distribution: C. mydas from Brazil (Werneck \& Silva, 2015) and Puerto Rico (Dyer et al., 1991; Dyer et al., 1995b).

Microscaphidium reticulare (Van Beneden, 1859) Looss, 1900 (Synonym: Microscaphidium caballeroi Groschaft, 1977, Microscaphidium japonicum Oguro, 1941 and Monostomum reticulare Van Beneden, 1859)

Site of infection: Small and large intestine.

Host and distribution: C. mydas from Brazil (Werneck \& Silva, 2015), Costa Rica (Santoro et al., 2006), Cuba (Groschaft et al., 1977) and Puerto Rico (Dyer et al., 1995b).

Microscaphidium warui Blair, 1986

Site of infection: Urinary bladder.

Host and distribution: C. mydas from Costa Rica (Santoro et al., 2006).

Microscaphidium aberrans Looss, 1902

Site of infection: Intestine.

Host and distribution: C. mydas from Panamá (Caballero, 1954)

Neoctangium travassosi Ruiz, 1943

[Synonym: Octangium travassosi (Ruiz, 1943) Yamaguti, 1958, Neoctangium trinidadi Gupta 1962]

Site of infection: Stomach and small and large intestine.

Host and distribution: E. imbricata from Puerto Rico (Fischthal \& Acholonu, 1976).
C. mydas from Brazil [Ruiz 1943 (see Muniz-Pereira et al., 2009); Travassos et al., 1969; Werneck \& Silva, 2015; Binoti et al., 2016)], Caribbean Sea (Gupta, 1962) and Trinidad (The West Indies) (Gupta, 1961).

Octangium hyphalum Blair, 1987

Site of infection: Intestine.

Host and distribution: C. mydas from Costa Rica (Santoro et al., 2006).

Octangium sagitta (Looss, 1899) Looss, 1902

[Synonym: Microscapha sagitta Looss, 1899, Octangium hasta Looss, 1902, Octangium takanoi Kobayashi, 1921 (see Blair, 1987)].

Site of infection: Stomach and small and large intestine.

Host and distribution: C. mydas from Puerto Rico (Dyer et al., 1991) and E. imbricata from Puerto Rico (Fischthal \& Acholonu, 1976; Dyer et al., 1995c).

Polyangium linguatula (Looss, 1899)

[Synonym: Microscaphidium linguatula Looss, 1899, Nephrobius colymbi Poche, 1926, Polyangium colymbi (Poche, 1926), Polyangium longiseminale Chattopadhyaya, 1972 and Polyangium miyajimai Kobayashi, 1915].

Site of infection: Small and large intestine.

Host and distribution: C. mydas from Brazil (Teixeira de Freitas \& Lent, 1938; Travassos et al., 1969; Werneck \& Silva, 2015; Binoti et al., 2016), Costa Rica (Santoro et al., 2006), Cuba (Groschaft et al., 1977) and Puerto Rico (Dyer et al., 1991; Dyer et al., 1995c).

\section{Superfamily Pronocephaloidea Looss, 1899 Family Pronocephalidae Looss, 1899}

Adenogaster serialis Looss, 1901

Site of infection: Small and large intestine.

Host and distribution: C. mydas from Panamá (Caballero et al., 1955) and Peru (Tantalean et al., 1992); E. imbricata from Costa Rica (Santoro et al., 2015) and Cuba (Vigueras, 1955) and L. olivacea from México (Pérez-Ponce de Leon et al., 1996; Vivaldo et al., 2006; Vivaldo et al., 2009).

Charaxicephaloides polyorchis Groschaft \& Tenora, 1978.

Site of infection: Stomach.

Host and distribution: C. mydas from Costa Rica (Santoro et al., 2009a) and Cuba (Groschaft \& Tenora, 1978; Groschaft et al., 1977).

Charaxicephaloides sp. Groschaft \& Tenora, 1978

Site of infection: Stomach.

Host and distribution: C. mydas from Costa Rica (Santoro et al., 2006). 
Charaxicephalus robustus Looss, 1901

Site of infection: Esophagus and stomach.

Host and distribution: C. mydas from Brazil (Binoti et al., 2016) and

Costa Rica (Santoro et al., 2006).

Cricocephalus americanus Vigueras, 1955

Site of infection: Stomach.

Host and distribution: E. imbricata from Cuba (Viegueras, 1955).

Cricocephalus albus (Kuhl \& Van Hasselt, 1822)

(Synonym: Cricocephalus delitescens Looss, 1899 and Cricocephalus koidzumii Kobayashi, 1921).

Site of infection: Esophagus, stomach and small intestine.

Host and distribution: C. mydas from Brazil (Ruiz, 1946; Travassos et al., 1969; Werneck \& Silva, 2015; Binoti et al., 2016), Costa Rica (Santoro et al., 2006), Panama (Caballero et al., 1955) and Trinidade (The West Indies) (Gupta, 1961); E. imbricata from Costa Rica (Santoro et al., 2015) and Puerto Rico (Fischthal \& Acholonu, 1976).

Cricocephalus megastomum Looss, 1902

Site of infection: Esophagus, stomach and small intestine.

Host and distribution: C. mydas from Brazil (Werneck \& Silva, 2015; Binoti et al., 2016), Costa Rica (Santoro et al., 2006) and Cuba (Groschaft et al., 1977); E. imbricata from Puerto Rico (Fischthal \& Acholonu, 1976; Dyer et al., 1995a).

Cricocephalus resectus Looss, 1902

Site of infection: Esophagus and stomach.

Host and distribution: C. mydas from Costa Rica (Santoro et al., 2006).

Cricocephalus vitallani (Gupta, 1962)

(Synonym: Neocricocephalus vitallani Gupta, 1962)

Site of infection: Intestine.

Host and distribution: C. mydas from Caribbean Sea (Gupta, 1962).

Desmogonius desmogonius Stephens, 1911

Site of infection: Esophagus, stomach and intestine.

Host and distribution: C. mydas from "Caribbean waters" (Coil \& Reid, 1965), Costa Rica. (Santoro et al., 2006) and Jamaica (Stephens, 1911).

Diaschistorchis pandus (Braun, 1901) Johnstone, 1913

Site of infection: Stomach and small intestine.

Host and distribution: C. mydas from Brazil (Werneck \& Silva, 2015); E. imbricata from Brazil (Werneck et al., 2015b), Cuba (Vigueras, 1955) and Puerto Rico (Fischthal \& Acholonu, 1976; Dyer et al., 1995a).
Metacetabulum invaginatum Teixeira de Freitas \& Lent 1938

Site of infection: Stomach and small intestine.

Host and distribution: C. mydas from Brazil (Teixeira de Freitas \& Lent, 1938; Ruiz, 1946; Travassos et al., 1969; Werneck \& Silva, 2015; Binoti et al., 2016); E. imbricata from Porto Rico (Fischthal \& Acholonu, 1976).

Pleurogonius americanus Caballero, Zerecero \& Grocott, 1955 [Synonym: Pyelosomum americanum (Caballero, Zerecero \& Grocott, 1955)]

Site of infection: Intestine.

Host and distribution: C. mydas from Panamá (Caballero et al., 1955).

Pleurogonius laterouteus Fischthal \& Acholonu, 1976

Site of infection: Large intestine.

Host and distribution: E. imbricata from Porto Rico (Fischthal \& Acholonu, 1976).

Pleurogonius linearis Looss, 1901

[Synonym: Pyelosomum lineare (Looss, 1901)]

Site of infection: Small and large intestine.

Host and distribution: C. mydas from Brazil (Ruiz, 1946; Travassos et al., 1969; Werneck \& Silva, 2015; Binoti et al., 2016) and Costa Rica (Santoro et al., 2006); E. imbricata from Costa Rica (Santoro et al., 2015), México (Caballero \& Zerecero, 1950) and Porto Rico (Fischthal \& Acholonu, 1976).

\section{Pleurogonius longiusculus Looss, 1901}

[Synonym: Pyelosomum longiusculum (Looss, 1901)]

Site of infection: Esophagus, stomach, small and large intestine. Host and distribution: C. mydas from Brazil (Ruiz, 1946; Travassos et al., 1969; Werneck \& Silva, 2015; Binoti et al., 2016), Costa Rica (Santoro et al., 2006) and Panamá (Caballero, 1954; Caballero et al., 1955).

Pleurogonius grocotti Caballero, 1954

Site of infection: Intestine.

Host and distribution: C. mydas from Panamá (Caballero, 1954).

Pleurogonius chelonii Mehra, 1939

(Synonym: Pleurogonius mehrai Ruiz, 1946).

Site of infection: Intestine.

Host and distribution: C. mydas from Trinidad (The West Indies) (Gupta, 1961).

Pleurogonius longibursatus Vigueras, 1955

[Synonym: Pyelosomum longibursatum (Vigueras, 1955)].

Site of infection: Intestine.

Host and distribution: E. imbricata from Cuba (Vigueras, 1955). 
Pleurogonius puertoricensis Fischthal \& Acholonu, 1976

Site of infection: Large intestine.

Host and distribution: E. imbricata from Puerto Rico (Fischthal \&

Acholonu, 1976).

Pleurogonius sindhii Mehra, 1939

[Synonym: Pyelosomum sindhii (Mehra, 1939)].

Site of infection: Intestine.

Host and distribution: C. mydas from Costa Rica (Santoro et al., 2006) and Panamá (Caballero et al., 1955).

\section{Pleurogonius solidus Looss, 1901}

[Synonym: Pyelosomum solidum (Looss, 1901); Glyphicephalus solidus Looss, 1901].

Site of infection: Intestine.

Host and distribution: C. mydas from Costa Rica (Santoro et al., 2006) and E. imbricata from Cuba (Vigueras, 1955).

Pleurogonius tortugueroi Santoro, Greiner, Morales \& Rodriguez-Ortíz, 2007.

Site of infection: Large intestine.

Host and distribution: C. mydas from Costa Rica (Santoro et al., 2007).

Pleurogonius trigonocephalus (Rudolphi, 1809) Looss, 1901.

[Synonym: Pyelosomum trigonocephalum (Rudolphi, 1809), and Monostoma trigonocephalum Rudolphi, 1809].

Site of infection: Esophagus, stomach, small intestine, large intestine and liver.

Host and distribution: C. mydas from Brazil (Travassos et al., 1969; Werneck \& Silva, 2015); E. imbricata from Costa Rica (Santoro et al., 2015), Cuba (Vigueras, 1955) and Puerto Rico (Fischthal \& Acholonu, 1976).

\section{Pleurogonius sp.}

Site of infection: Intestine.

Host and distribution: C. mydas from British West Indies (Greiner et al., 1980) and Costa Rica (Santoro et al., 2006).

\section{Pleurogonius lobatus (Looss, 1901)}

(Synonym: Glyphicephalus lobatus Looss, 1901).

Site of infection: Stomach and small and large intestine.

Host and distribution: C. mydas from Brazil (Ruiz, 1946; Travassos et al., 1969; Werneck \& Silva, 2015; Binoti et al., 2016), Costa Rica (Santoro et al., 2006), Panama (Caballero et al., 1955), and Puerto Rico (Dyer et al., 1991); E. imbricata from Costa Rica (Santoro et al., 2015) and Puerto Rico (Fischthal \& Acholonu, 1976); L. olivacea from México (Pérez-Ponce de Leon et al., 1996).

Pleurogonius latus Fischthal \& Acholonu 1976 (Synonym: Glyphicephalus latus Fischthal \& Acholonu 1976).
Site of infection: Small intestine (occasionally stomach and large intestine)

Host and distribution: E. imbricata from Puerto Rico (Fischthal \& Acholonu, 1976).

Pleurogonius stenobursatum (Fischthal \& Acholonu, 1976) Blair, 2005

[Synonym: Pyelosomum stenobursatum (Fischthal \& Acholonu, 1976) Pérez ponce de León \& Brooks, 1995, and Epibathra stenobursata Fischthal \& Acholonu 1976].

Site of infection: Large intestine.

Host and distribution: E. imbricata from Puerto Rico (Fischthal \& Acholonu, 1976).

Pronocephalus obliquus Looss, 1899

(Synonym: Pronocephalus mehrai Chattopadhyaya, 1972).

Site of infection: Esophagus, stomach, small intestine, large intestine and liver.

Host and distribution: C. mydas from Brazil (Travassos et al., 1969; Werneck \& Silva, 2015; Binoti et al., 2016) and Costa Rica (Santoro et al., 2006); E. imbricata from Brazil (Werneck et al., 2015b).

\section{Pronocephalus trigonocephalus Looss, 1899}

Site of infection: Esophagus, stomach and intestine.

Host and distribution: C. caretta from Brazil (Viana, 1924); C. mydas from Brazil (Ruiz, 1946; Travassos et al., 1969; Binoti et al., 2016) and Panama (Caballero et al., 1955).

\section{Pyelosomum cochlear Looss, 1899}

Site of infection: Urinary bladder.

Host and distribution: C. mydas from Brazil (Werneck \& Silva, 2015; Binoti et al., 2016), Costa Rica (Santoro et al., 2006), Panamá (Caballero, 1954) and Puerto Rico (Dyer et al., 1995b; Dyer et al., 1991); L. olivacea from Brazil (Werneck et al., 2015c).

Pyelosomum crassum (Looss, 1901) Ruiz, 1946

(Synonym: Glyphicephalus crassa Looss, 1901).

Site of infection: Small and large intestine.

Host and distribution: C. mydas from Brazil (Ruiz, 1946; Travassos et al., 1969; Werneck \& Silva, 2015), and E. imbricata from Costa Rica (Santoro et al., 2015).

Pyelosomum posterorchis Oguro, 1936

Site of infection: Small intestine.

Host and distribution: C. mydas from Panama (Caballero et al., 1955); E. imbricata from Costa Rica (Santoro et al., 2015) and Puerto Rico (Fischthal \& Acholonu, 1976).

Pyelosomum renicapite (Leidy, 1856)

[Synonym: Astrorchis renicapite (Leidy, 1856), Monostomum nephrocephalum Diesing, 1858, Monostomum sphargidis MacCallum, 1921 and Pyelosomum longicaecum Luhman, 1935] 
Site of infection: Small and large intestine.

Host and distribution: C. caretta from Brazil (Werneck et al., 2008a); D. coriacea from Brazil and Uruguay (Werneck et al., 2012) and Puerto Rico (Dyer et al., 1995b); L. olivacea from México (Pérez-Ponce de Leon et al., 1996).

Rameshwarotrema uterocrescens Rao, 1975

Site of infection: Esophagus, stomach, small and large intestine. Host and distribution: C. mydas from Brazil (Werneck \& Silva, 2015) and Costa Rica (Santoro et al., 2006); E. imbricata from Puerto Rico (Dyer et al., 1995a).

Ruicephalus minutus (Ruiz, 1946) Skrjabin, 1955

(Synonym: Pronocephalus minutus Ruiz, 1946)

Site of infection: Stomach and small intestine.

Host and distribution: C. mydas from Brazil (Ruiz, 1946; Travassos et al., 1969; Werneck \& Silva, 2015; Binoti et al., 2016).

\section{Suborder Xiphidiata Olson Cribb, Tkach, Bray \& Littlewood,} 2003

Superfamily Gorgoderoidea Looss, 1901

Family Gorgoderidae Looss, 1899

Subfamily Anaporrhutinae Looss, 1901

Plesiochorus cymbiformis (Rudolphi, 1819) Looss, 1901

[Synonym: Phyllodistomum cymbiforme (Rudolphi, 1819; Plesiochorus cymbiformis elongatus Pigulevsky, 1953]

Site of infection: Urinary bladder and small intestine.

Host and distribution: C. mydas from Brazil (Binoti et al., 2016) and Panamá (Caballero, 1954); E. imbricata from Puerto Rico (Fischthal \& Acholonu, 1976); L. olivacea from Costa Rica (Santoro \& Morales, 2007).

\section{Superfamily Microphalloidea Ward, 1901}

Family Pachypsolidae Yamaguti, 1958

Pachypsolus irroratus (Rudolphi, 1819) Looss, 1902

[Synonym: Pachypsolus branchus Barker, 1922, Pachypsolus lunatus Looss, 1901, Pachypsolus puertoricensis Fischthal e Acholonu 1976 and Pachypsolus ovalis Linton, 1910)].

Site of infection: Stomach and intestine.

Host and distribution: C. mydas from Panamá (Caballero et al., 1955); E. imbricata from Porto Rico (Fischthal \& Acholonu, 1976); L. olivacea from México (Pérez-Ponce de Leon et al., 1996) and Costa Rica (Santoro \& Morales, 2007).

\section{Superfamily Plagiorchioidea Lühe, 1901}

Family Brachycoeliidae Looss, 1899

Cymatocarpus solearis (Braun, 1899) Braun, 1901

(probably Synonym: Cymatocarpus undulatus Looss, 1899)

Site of infection: Esophagus, stomach and small intestine.

Host and distribution: C. mydas from Brazil (Werneck \& Silva, 2015) and Mexico (Caballero, 1959); E. imbricata from Brazil (Werneck et al., 2015b)
Family Plagiorchiidae Lühe, 1901

Enodiotrema megachondrus (Looss, 1899) Looss, 1901

Site of infection: Small intestine.

Host and distribution: C. mydas from Brazil (Werneck et al., 2016a); E. imbricata from Cuba (Groschaft et al., 1977); L. olivacea from Costa Rica (Santoro \& Morales, 2007) and México (Pérez-Ponce de Leon et al., 1996; Vivaldo et al., 2006).

Enodiotrema reductum Looss, 1901

Site of infection: Small intestine.

Host and distribution: C. mydas from Panamá (Caballero, 1954); E. imbricata from Costa Rica (Santoro et al., 2015) and Puerto Rico (Fischthal \& Acholonu, 1976; Dyer et al., 1995a).

\section{Family Styphlotrematidae Baer, 1924}

Styphlotrema solitaria (Looss, 1899) Odhner, 1911

Site of infection: Esophagus, stomach and small intestine.

Host and distribution: E. imbricata from Brazil (Werneck \& Silva, 2012), Cuba (Groschaft et al., 1977) and Puerto Rico (Fischthal \& Acholonu, 1976).

\section{Family Telorchiidae Looss, 1899}

Orchidasma amphiorchis (Braun, 1899)

Site of infection: Stomach and small intestine.

Host and distribution: C. caretta from Argentina (Boero \& Led, 1974) and Brazil (Werneck et al., 2008a); C. mydas from Brazil (Teixeira de Freitas \& Lent, 1938; Travassos et al., 1969; Binoti et al., 2016), México (Caballero \& Zerecero, 1950; Caballero, 1962); Panama (Caballero et al., 1955), and Peru (Tantalean et al., 1992); E. imbricata from Puerto Rico (Fischthal \& Acholonu, 1976); L. olivacea from México (Pérez- Ponce de Leon et al., 1996).

Order Diplostomida Olson, Cribb, Tkach, Bray \& Littlewood, 2003

Suborder Diplostomata Olson, Cribb, Tkach, Bray \& Littlewood, 2003

Superfamily Schistosomatoidea Stiles \& Hassall, 1898

\section{Family Spirorchiidae Stunkard, 1921}

Amphiorchis amphiorchis Price, 1934

Site of infection: Blood vessels of large intestine.

Host and distribution: E. imbricata from Puerto Rico (Fischthal \& Acholonu, 1976).

\section{Amphiorchis caborojoensis Fischthal \& Acholonu, 1976}

Site of infection: Blood vessels of lung, liver, small intestine, body wash and heart.

Host and distribution: E. imbricata from Puerto Rico (Dyer et al., 1995a; Fischthal \& Acholonu, 1976) and Brazil (Werneck et al., 2008b; Dutra et al., 2012; Werneck et al., 2015b). 
Amphiorchis indicus Mehrotra, 1973

Site of infection: Esophagus, stomach, small intestine, large intestine and liver.

Host and distribution: C. mydas from Brazil (Werneck \& Silva, 2013; Werneck \& Silva, 2015).

Amphiorchis solus (Simha \& Chattopadhyaya, 1970) Platt, 2002 (Synonym: Squaroacetabulum solus Simha \& Chattopadhyaya, 1970)

Site of infection: Intestine and heart.

Host and distribution: C. mydas from Brazil (Werneck et al., 2011; Werneck \& Medeiros, 2016) and Costa Rica (Santoro et al., 2006).

Carettacola stunkardi (Martin \& Bamberger, 1952)

[Synonym: Haemoxenicon stunkardi Martin \& Bamberger, 1951,Carettacola chelonenecon (Martin \& Bamberger, 1952), and Haemoxenicon chelonenecon Martin \& Bamberger, 1952)].

Site of infection: Blood vessels of urinary bladder, heart, body wash and liver.

Host and distribution: C. mydas from Brazil (Werneck et al., 2013) and Panamá (Caballero et al., 1955); E. imbricata from Brazil (Werneck et al., 2008b; Werneck et al., 2015b) and Costa Rica (Santoro et al., 2015).

Hapalotrema postorchis Rao, 1976

Site of infection: Great vessels and heart.

Host and distribution: C. mydas from Brazil (Werneck et al., 2015d) and Costa Rica (Santoro et al., 2006); E. imbricata from Brazil (Werneck et al., 2014).

Hapalotrema synorchis Luhman, 1935

Site of infection: Heart.

Host and distribution: E. imbricata from Puerto Rico (Fischthal \& Acholonu, 1976).

Learedius learedi Price, 1934

Site of infection: Esophagus, stomach, small and large intestine, liver, gall bladder, heart, lung, body wash, kidney.

Host and distribution: C. mydas from Bermuda (Rand \& Wiles, 1985), Brazil (Werneck et al., 2006; Werneck \& Silva, 2015; Binoti et al., 2016), British West Indies (Greiner et al., 1980), Costa Rica (Santoro et al., 2006), Panama (Caballero et al., 1955), Puerto Rico (Dyer et al., 1991), México (Cordeiro-Tapia et al., 2004; Inohuye-Rivera et al., 2004); E. imbricata from Puerto Rico (Dyer et al., 1995a).

Learedius orientalis Mehra, 1939

Site of infection: Heart.

Host and distribution: C. mydas from Puerto Rico (Dyer et al., 1995a); E. imbricata from Puerto Rico (Fischthal \& Acholonu, 1976).
Monticellius indicum Mehra, 1939

Site of infection: Heart.

Host and distribution: C. mydas from Brazil (Werneck et al., 2008c; Werneck \& Silva, 2015 and Costa Rica (Santoro et al., 2006; Santoro et al., 2009b); E. imbricata from Brazil (Werneck et al., 2015e).

Neospirorchis schistosomatoides Price 1934

Site of infection: Heart chambers and Aorta.

Host and distribution: C. mydas from Bermuda (Rand \& Wiles, 1985) and Brazil (Werneck et al., 2016b).

\section{Neospirorchis sp.}

Site of infection: Small intestine, liver, heart, lung, body wash. Host and distribution: C. mydas from Brazil (Werneck \& Silva, 2015).

\section{Phylum Nematoda Rudolphi, 1808 \\ Class Chromadorea Inglis, 1983 \\ Subclass Chromadoria Adamson, 1987 \\ Order Rhabditida Chitwood, 1933 \\ Suborder Spirurina Railliet \& Henry, 1915 \\ Infraorder Ascaridomorpha De Ley \& Blaxter 2002 \\ Superfamily Ascaridoidea Baird, 1853 \\ Family Anisakidae Skrjabin e Karokhin, 1945}

Sulcascaris sulcata (Rudolphi, 1819)

Site of infection: Esophagus, stomach and small intestine.

Host and distribution: C. caretta from Brazil (Werneck et al., 2008a) and Uruguay (Lent \& Teixeira de Freitas, 1948); C. mydas from Brazil (Teixeira de Freitas \& Lent, 1946).

\section{Anisakis larvae}

Site of infection: Body wash.

Host and distribution: E. imbricata from (Werneck et al., 2015b).

\section{Superfamily Cosmocercoidea Travassos, 1935 Family Kathlaniidae Travassos, 1918}

Kathlania leptura (Rudolphi, 1819)

Site of infection: Small and large intestine.

Host and distribution: C. caretta from Brazil (Werneck et al., 2008a); C. mydas from Brazil (Travassos, 1918).

Tonaudia freitasi Vicente \& Santos, 1968.

Site of infection: Stomach.

Host and distribution: C. mydas from Brazil (Vicente \& Santos, 1968).

\section{Nematode larvae}

Site of infection: Esophagus, stomach, small and large intestine, liver.

Host and distribution: C. mydas from Brazil (Werneck \& Silva, 2015). 
Host-Parasite list

Chelonia mydas

\section{Digeneans}

Family Calycodidae

- Calycodes anthos

\section{Family Rhytidodidae}

- Rhytidodes gelatinosus

- Rhytidodoides intestinalis

- Rhytidodoides similis

\section{Family Cladorchiidae}

Schizamphistomum erratum

Schizamphistomum scleroporum

Schizamphistomoides spinulosum

\section{Family Microscaphidiidae}

- Angiodictyum longum

- Angiodictyum parallelum

- Deuterobaris chelonei

- Deuterobaris intestinalis

- Deuterobaris proteus

- Microscaphidium aberrans

- Microscaphidium reticulare

- $\quad$ Microscaphidium warui

- Neoctangium travassosi

- Octangium hyphalum

- Octangium sagitta

- Polyangium linguatula

\section{Family Pronocephalidae}

- Adenogaster serialis

- Charaxicephaloides polyorchis

- Charaxicephalus robustus

- Cricocephalus albus

- Cricocephalus megastomum

- Cricocephalus resectus

- Cricocephalus vitallani

- Desmogonius desmogonius

- Diaschistorchis pandus

- Metacetabulum invaginatum

- Pleurogonius americanus

- Pleurogonius chelonii

- Pleurogonius grocotti

- Pleurogonius linearis

- Pleurogonius longiusculus

- Pleurogonius sindhii
- Pleurogonius solidus

- Pleurogonius tortugueroi

- Pleurogonius trigonocephalus

- Pleurogonius lobatus

- Pronocephalus obliquus

- Pronocephalus trigonocephalus

- Pyelosomum cochlear

- Pyelosomum crassum

- Pyelosomum posterorchis

- Rameshwarotrema uterocrescens

- Ruicephalus minutus

Family Gorgoderidae

- Plesiochorus cymbiformis

Family Pachypsolidae

- Pachypsolus irroratus

Family Brachycoeliidae

- $\quad$ Cymatocarpus solearis

Family Plagiorchiidae

- Enodiotrema reductum

Family Telorchiidae

- $\quad$ Orchidasma amphiorchis

Family Spirorchiidae

- Amphiorchis indicus

- Amphiorchis solus

- Carettacola stunkardi

- Hapalotrema postorchis

- Learedius learedi

- Learedius orientalis

- Monticellius indicum

- Neospirorchis schistosomatoides

\section{Nematodes}

FamilyAnisakidae

- $\quad$ Sulcascaris sulcata

\section{Family Kathlaniidae}

- Kathlania leptura.

- Tonaudia freitasi 


\section{Eretmochelys imbricata}

\section{Digeneans}

Family Calycodidae

- Calycodes caborojoensis

\section{Familia Rhytidodidae}

- Rhytidodes gelatinosus

\section{Family Cladorchiidae}

- Schizamphistomum scleroporum

\section{Family Microscaphidiidae}

- Angiodictyum anteroporum

- Angiodictyum mooreae

- Angiodictyum parallelum

- Neoctangium travassosi

- Octangium sagitta

\section{Family Pronocephalidae}

- Adenogaster serialis

- Cricocephalus albus

- Cricocephalus americanus

- Cricocephalus megastomum

- Diaschistorchis pandus

- Metacetabulum invaginatum

- Pleurogonius laterouteus

- Pleurogonius latus

- Pleurogonius linearis

- Pleurogonius lobatus

- Pleurogonius longibursatus

- Pleurogonius puertoricensis

- Pleurogonius solidus

- Pleurogonius trigonocephalus

- Pleurogonius stenobursatum

- Pronocephalus obliquus

- Pyelosomum crassum

- Pyelosomum posterorchis

- Rameshwarotrema uterocrescens

\section{Family Gorgoderidae}

- Plesiochorus cymbiformis

\section{Family Pachypsolidae}

- Pachypsolus irroratus

\section{Family Brachycoeliidae}

$$
\text { - Cymatocarpus solearis }
$$

Family Plagiorchiidae

- Enodiotrema megachondrus

- Enodiotrema reductum

Family Styphlotrematidae

- Styphlotrema solitaria

Family Telorchiidae

- Orchidasma amphiorchis

Family Spirorchiidae

- Amphiorchis amphiorchis

- Amphiorchis caborojoensis

- Carettacola stunkardi

- Hapalotrema postorchis

- Hapalotrema synorchis

- Learedius learedi

- Learedius orientalis

- Monticellius indicum

Nematodes

FamilyAnisakidae

- Anisakis larvae

Lepidochelys olivacea

Digeneans

Family Calycodidae

- Calycodes anthos

Family Sclerodistomidae

- Prosorchis psenopsis

Family Pronocephalidae

- Adenogaster serialis

- Pleurogoius lobatus

- Pyelosomum cochlear

- Pyelosomum renicapite

Family Gongoderidae

- Plesiochorus cymbiformis

Family Pachypsolidae

- Pachypsolus irroratus

Family Plagiorchiidae

- Enodiotrema megachondrus

Family Telorchiidae

- Orchidasma amphiorchis 


\section{Caretta caretta}

\section{Aspidogastrids \\ Family Aspidogastridae \\ - Lophotaspis vallei}

\section{Digeneans \\ Family Calycodidae \\ - Calycodes anthos}

\section{Familia Rhytidodidae \\ - Rhytidodes gelatinosus}

\section{Family Pronocephalidae}

- $\quad$ Pronocephalus trigonocephalus

- Pyelosomum renicapite

\section{Family Telorchiidae \\ - Orchidasma amphiorchis}

\section{Nematodes \\ FamilyAnisakidae \\ - Sulcascaris sulcata \\ Family Kathlaniidae \\ - Kathlania leptura}

Dermochelys coriacea

\section{Digeneans \\ Family Calycodidae \\ - Calycodes anthos}

\section{Family Pronocephalidae \\ - Pyelosomum renicapite}

\section{Discussion}

This checklist demonstrates the occurrence of 79 taxa of helminthes parasites. Seventy six represent the phylum Platyhelminthes (one from the order Aspidogastrida, 11 from the order Diplostomida and 64 from the order Plagiorchiida) represented by 14 families. Three represent the phylum Nematoda,

Five species of sea turtles (C. mydas, C. caretta, E. imbricata, $L$. olivacea and $D$. coriacea) had records of helminths in the $\mathrm{Ne}-$ otropical region. The host with the largest number of records of parasites was C. mydas (records of only the genus were excluded from the calculation), representing 62 parasite species, followed by $E$. imbricata (42 species), L. olivacea (10 species), C. caretta (8 species) and $D$. coriacea (2 species). Overall helminths are reported in 12 countries and three are reported for the region in which the present study took place (i.e. the Caribbean Sea).

Among the countries in which helminths are reported, Brazil, Costa Rica and Panamá have the largest number of records for $C$. mydas. Lepidochelys olivacea is the best studied sea turtle from Mexico, with eight records of parasite species. Porto Rico stands out for the large number of parasite species reported in $E$. imbricata, such as $C$. caborojoensis, $A$. anteroporum, $A$. mooreae, $P$. laterouterus, $P$. puertoricensis, $P$. latus, $P$. sternobursatum, $A$. amphiorchis and $H$. synorchis, which are only found in this country in this region.

Dermochelys coriacea had the fewest reports of parasites from the region with only $C$. anthos and $P$. renicapite) being detected. This species of turtle has been studied the least of all marine turtles regarding its parasites around the world (see Werneck et al., 2012). This paper presents the largest compilation of data on helminth parasites in sea turtles in the Neotropical region published to date.

\section{Acknowledgements}

The authors wish to express their special thanks to Dr. Paula Baldassin, Daniel Clifton and the reviewers for critical reading and suggestions to improve the manuscript.

\section{References}

ARAúso, T.L. (1941): Nota sobre um trematoide Aspidogastridae de tartaruga marinha. Bol. Ind. Anim., 4: 3 - 4

Binoti, E., Gomes, M.C., de Calais Junior, A., Werneck, M.R., MarTINS, I.V.F. BoELoNI, J.N. (2016): Helminth fauna of Chelonia mydas (Linnaeus, 1758) in the south of the State of Espirito Santo and description of tissue injury. Helminthologia, 53(2): 195 - 199. DOI: 10.1515/helmin-2016-0012

BLAIR, D. (1987): A revision of the subfamily Octangiinae (Platyhelminthes: Digenea: Microscaphidiidae) parasitic in marine turtles (Reptilia: Chelonia). Aust. J. Zool. 35: 75 - 92

BoERO, J.J., LED, J.E. (1974): Parasites of the authochthonous fauna. Rev. de Agronomia y de Veterinária, 3: 16 - 17

Caballero, C.E. (1954): Helmintos de la República de Panamá. X. Algunos tremátodos de Chelonia mydas (L.) tortuga marina comestible del Océano Pacífico del norte. An. Inst. Biol. Univ. Nac. Auton. Mex., 8: $31-58$

Caballero, E.C. (1959): Trematodos de las tortugas de Mexico VII. Description de un trematodo digeneo que parasita a tortugas marinas comestibles del puerto de Acaplco, Guerrero. An. Inst. Biol./ Univ. Nac. Auton. Mex., 33: 159 - 166

Caballero, C.E. (1962): Trematodos de las tortugas de México. X Presencia de Orchidasma amphirochis (Braun, 1899) Looss, 1900 en una tortuga marina, Chelone mydas de las Costas del estado de Tamaulipas, México. An. Inst. Biol./ Univ. Nac. Auton. Mex., 33: $47-55$

Caballero, C.E., Zerecero M.C. (1950): Trematodos de las tortugas de Mexico VI. Ver. Med. Vet. Y Paras., 9: 123 - 132 
Caballero, C.E., Zerecero M.C., Grocott, R.G. (1955): Helmintos de la República de Panamá. XI. Tremátodos de Chelonia mydas (L) tortuga marina comestible del Océano Pacífico del norte. $2^{\mathrm{a}}$ parte. An. Inst. Biol./ Univ. Nac. Auton. Mex., 26: 149 - 191

ColL, W.H., ReID, W.A. (1965): Desmogonius desmogonius Stephens, 1911 (pronocephalidae: Digenea), a redescription with observations on egg filament formation. Z. Parasitenkd., 25: 506-509 Cordeiro-Tapia, A.A., Gardner, S.C., Arellano-Blanco, J., InoHUYE-RIVERA, R.B. (2004): Learedius learedi infection in black turtles (Chelonia mydas agassizii), Baja California sur, Mexico. J. Parasitol., 90(3): 645 - 647. DOI: 10.1645/GE-165R

Dutra, G.H.P., Silva, A.N., Nascimento, C.L., Wernck, M.R. (2012): Lesões macroscópicas e histopatológicas da infecção por helmintos da Family Spirorchiidae em Eretmochelys imbricata Linnaeus 1758 (Testudines, Chelonidae): relato de um caso no litoral brasileiro. Natural Resources, Aquidabã, 2(1): 83 - 89. DOI: 10.6008/ESS2237-9290.2012.001.0006

Dyer, W.G., Williams, E.H.J., BunkLey-Williams, L. (1995c): Angiodictyum mooreae n.sp. (Digenea: Microscaphidiidae) and Other digeneas from na Atlântic Hawksbill turtle Eretmochelys imbricata from Puerto Rico. J. Aquat. Anim. Health, 7(1): 38 - 41. DOI: 10.1577/1548-8667(1995)007<0038:AMNSDM>2.3.CO;2

Dyer, W.G., Williams, E.H.J., BunkLeY-Williams, L (1995b): Digenea of the green turtle (Chelonia mydas) and the leatherback turtle (Dermochelys coriacea) from Puerto Rico. Caribb. J. Sci., 31(3-4): $269-273$

Dyer, W.G., Williams, E.H.J., BunkLey-Williams, L. (1991): Some Digeneans (Trematoda) of the green turtle, Chelonia mydas (Testudines: Cheloniidae) from Puerto Rico. Proc. Helminthol. Soc. Wash., 58(2): 176 - 180

Dyer, W.G., Williams, E.H.J. Bunkley-Williams, L., Moore, D. (1995a): Some digeneans (Trematoda) of the hawksbill turtle, Eretmochelys imbricata imbricata (Testudines: Cheloniidae) from Puerto Rico. Proc. Helminthol. Soc. Wash., 62: 13 - 17

Fischthal, J.H., Acholonu, A.D. (1976) Some digenetic trematodes from the Atlantic hawksbill turtle, Eretmochelys imbricate imbricata (L) from Puerto Rico. Proc. Helminthol. Soc. Wash., 43(2): 174 - 185 Greiner, E.C., Forrester, D.J., Jacobson, R. (1980): Helminthis of mariculture-reared green turtles (Chelonia mydas mydas) from Grand Cayman, British West Indies. Proc. Helminthol. Soc. Wash., 47(1): $142-144$

Groschaft, J., TenORA, F. (1978): Charaxicephaloides polyorchis Gen Nov, Sp Nov (Trematoda: Charaxicephalinae) from Chelonia mydas mydas (Testudinata) in Cuba. Vestnik Cs. Spol. Zool., 42(2): $108-111$

Groschaft, J., Otero, A.C., Tenora, F. (1977): Trematodes (Trematoda) from Cuban turtles Chelonia mydas mydas (L) and Eretmochelys imbricata imbricata (L) Testudinata-Cheloniidae). Acta Univ. Agriculturae, 25(4): 155 - 167

GUPTA, S.P. (1961): On some trematodes from the intestine of the marine turtle, Chelone mydas from the Caribbean Sea. Can. $j$. zool., 39: $293-298$
GUPTA, S.P. (1962): On two new trematodes from the intestine of the marine turtles, Chelone mydas, from the Caribbean Sea. Indian j. Helminthol., 14(2): $71-76$

Inohuye-Riveira, R., Cordeiro-Tapia, A., Arellano-Blanco, J., GARDNER, S.C. (2004): Learedius learedi Price, 1934 (Trematoda: Spirorchiidae), in black turtle (Chelonia mydas agassizii) hearts from Magdalena Bay, Baja California Sur, Mexico. Comp. Parasitol., 71(1): 37 - 41.DOI: 10.1654/4113

IUCN- International Union for Conservation of Nature (2015): Red List of Threatened Species. Version 2015-4. Retrieved March 21, from: www.iucnredlist.org

Lent, H., Teixeira de Freitas, J.F. (1948): Uma coleção de nematódeos, parasitos de vertebrados do Museu de História natural de Montevidéu. Mem. Inst. Oswaldo Cruz, 46(1), 1 - 71

LuTz, P.L., Musick, J.A. (1996): The biology of sea turtles. Washington, DC, CRC Press, $446 \mathrm{pp}$.

Muniz-PereiRA, L.C., VieIRA, F.M., Luque, J.L. (2009): Checklist of helminth parasites of threatened vertebrate species from Brazil. Zootaxa, 2123, 1 - 45

Pérez-Ponce de León, G. P., García-Prieto, L., León-Règagnon, V. (1996): Gastrointestinal Digenetic trematodes of olive ridley's turtle (Lepidochelysolivacea) from Oaxaca México. Taxonomy and infracommunity structure. J. Helminthol. Soc. Wash., 63(1): 76 - 82 RAND, T.G., WILES, M. (1985): Histopathology of infection by Learedius learedi Price, 1934 and Neospirorchis schistomatoides Price, 1934 (Digenea: Spirorchiidae) in Wild Green turtles, Chelonia mydas L., from Bermuda. J. Wildl. Dis., 21(4): 461 - 463

Ruiz, J.M. (1943): Neoctangium travassosi gen. N., sp. (Trematoda: Paramphistomoidea) parasito de quelônio marinho. Chave dos gêneros da Family Microscaphidiidae Travassos, 1922. Memo. Inst. Butantan, 17: 35 - 45

RuIz, J.M. (1946): Pronocephalidae (Trematoda) Estudo das espécies brasileiras e revisão da Família. Memo. Inst. Butantan, 19: $249-372$

Santoro, M., Brandmayr, O., Greiner, E.C., Morales, J.A., Rodrigues-Ortiz, B. (2009a): Redescription of Charaxicephaloides polyorchys Groschaft ant Tenora 1978 (Digenea: Pronocephalidae) from the Green turtle Chelonia mydas in Costa Rica. Helminthologia, 46(2): 97 - 99. DOI: 10.2478/s11687-009-0019-6

Santoro, M., Greiner, E.C., Morales, J.A., Rodrígues-Ortiz, B. (2007): A new pronocephalid, Pleurogonius tortugueroi n. sp. (Digenea), from the intestine of Green sea turtles (Chelonia mydas) in Costa Rica. Parassitologia, 49(1-2): 97 - 100

Santoro, M., Greiner, E.C., Morales, J.A., Rodrigues-Ortiz, B. (2006): Digenetic trematode community in nesting green sea turtles (Chelonia mydas) from Tortuguero National Park, Costa Rica. J. Parasitol., 92(6): 1202 - 1206. DOI: 10.1645/GE-866R.1

Santoro, M., Greiner, E.C., Morales, J.A., Rodrigues-Ortiz, B. (2009b): Redescription of Monticellius indicum Mehra, 1939 (Digenea: Spirorchiidae) from the heart of Green Sea Turtle (Chelonia mydas) in Costa Rica. Open Parasitol J., 3: 4 - 8

SANTORO, M., Morales, J.A. (2007): Some digenetic trematodes of 
the olive ridley sea turtle, Lepidochelys olivacea (Testudines, Cheloniidae) in Costa Rico. Helminthologia, 44(1): 25 - 28

Santoro, M., Morales, J.A., Bolaños, F., Chaves, G., De Stefano, M. (2015): Helminths of hawksbill turtle (Eretmochelys imbricata) from the Pacific coast of Costa Rica. Helminthologia, 52(1): 67 70. DOI: $10.1515 /$ helmin-2015-0012

Stephens, J.W.W. (1911): Desmogonius desmogonius, a new species and genus of monostome flukes. Ann. Trop. Med. Parasit., 5: $497-500$

Tantaleán, M., Sarmiento, L., Huiza, A. (1992): Digeneos (Trematoda) del Perú. Boletín de Lima (Perú),14: 47 - 84

TeixeIRA de FreitAs, J.T., Lent, H. (1946): "Porrocaecum sulcatum" (Rudolphi, 1819). Rev. Bras. Biol., 6(2): 235 - 238

TeIXEIRA DE FreItAS, J.T., LeNT, H. (1938): Sobre alguns trematodeos parasitos de Chelone mydas (L.), principalmente Paramphistomoides. Mem. Inst. Oswaldo Cruz, 33(1): 79 - 97

Travassos, L. (1918): Informações sobre a familia Kalhlanidae $n$. nom. Rev. Bras. Biol., , 2: $83-88$

Travassos, L., Freitas, T., Kohn, A. (1969): Trematódeos do Brasil. Mem. Inst. Oswaldo Cruz, 67: 1 - 886

VIANA, L. (1924): Tentativa de catalogação das espécies brasileiras de trematódeos. Mem. Inst. Oswaldo Cruz, 17: 95 - 227

Vicente, J.J., Santos, E. (1968): Terceira espécie do gênero "Tonaudia" Travassos, 1918, (Nemaloda, Kathlaniidae). Atas Soc. Biol. Rio J., 12(2): $55-56$

Vigueras, P. (1955): Contribución al conocimento de la fauna helmintológica cubana. Mem. Soc. Cub. Hist. Nat., 22: 21 - 71

Vivaldo, S.G., Márquez, L.J.G., Sarabia, D.O., García, J.L.V., Casa, F.C. (2009): Pathology in the Olive Ridley turtles (Lepidochelys olivacea) that arrived to the shores of Cuyutlan, Colima, Mexico. Vet. Mex., 40: 69 - 78

Vivaldo, S.G., Sarabia, D.O., Salazar, C.P., Hernándz, A.G., Lezama, J.R. (2006): Identificación de parásitos y epibiontes de la tortuga Golfi na (Lepidochelys olivacea) que arribó a playas de Michoacán y Oaxaca, México. Vet. Mex., 37(4): $431-440$

Werneck, M.R., Amorim, D.B., Blair, D. (2015c): Olive Ridley Sea Turtle Lepidochelys olivacea (Eschscholtz, 1829) from Brazil as a New Host of Pyelosomum cochlear Looss 1899 (Digenea: Pronocephalidae). Comp. Parasitol., 82(1): 144 - 147. DOI: 10.1654/4739.1

Werneck, M.R., Baldassin, P., D’Azeredo, F., Trazzi, A., Berger, B.C. (2014): The Hawksbill Sea Turtle Eretmochelys imbricata Linnaeus 1758 (Testudines, Cheloniidae) as new host of Hapalotrema postorchis Rao, 1976 (Digenea: Spirorchiidae). Comp. Parasitol., 81(1): 75 - 78. DOI: http://dx.doi.org/10.1654/4662.1

Werneck, M.R., Baldassin, P., Torres, F., Trazzi, A., Berguer, B. (2013): Report of Carettacola stunkardi (Martin \& Bamberger, 1952) Dailey, Fast \& Balazs, 1991 (Digenea: Spirorchiidae) infecting Green turtle Chelonia mydas Linnaeus, 1758 (Testudines, Cheloniidae) in Brazil. Braz. J. Biol., 73(3): 675 - 676

Werneck, M.R., Becker, J.H., Gallo, B.M.G., Silva, R.J. (2006): Learedius learedi Price 1934 (Digenea, Spirorchiidae) in Chelonia mydas Linnaeus 1758 (Testudines, Chelonidae) in Brazil: case report. Arq. Bras. Med. Vet. Zootec., 58(4): 550 - 555

Werneck, M.R., Binoti, E., Martins, I.V.F., Calais Júnior, A., Gomes, M.C., Boeloni, J.N., Trazzl, A., Berguer, B.C. (2015a): Occurrence of Rhytidodoides similis Price, 1939 (Digenea: Rhytidodidae) and lesions due to spirorchiid eggs in a Green Turtle Chelonia mydas Linnaeus, 1758 (Testudines, Cheloniidae) from Brazil. Comp. Parasitol., 82(2): 291 - 295. DOI: 10.1654/4747.1 Werneck, M.R., Conti, L.M., Berger, B. (2016a). Report of Enodiotrema megachondrus (Looss, 1899) Looss, 1901 (Digenea: Plagiorchiidae) in a green turtle Chelonia mydas Linnaeus, 1758 (Testudines, Cheloniidae) from Brazil. Helminthologia. Advance online publication. DOI 10.1515/helmin-2016-0019

WerneCK, M.R., Gallo, B.M.G., Lima, E.H.S.M., Silva, R.J. (2011): Occurrence of Amphiorchis solus Simha \& Chattopadhyaya, 1970 (Digenea, Spirorchiidae) infecting Green turtle Chelonia mydas Linnaeus, 1758 (Testudines, Cheloniidae) in Brazil. Comp. Parasitol., 78(1): 200 - 203. DOI: 10.1654/4435.1

Werneck, M.R., Gallo, B.M. G., Silva, R.J. (2008c): First report of Monticellius indicum Mehra, 1939 (Digenea: Spirorchiidae) infecting Chelonia mydas Linnaeus, 1758 (Testudines: Chelonidae) from Brazil. Braz. J. Biol., 68(2): 455 - 456

Werneck, M.R., Gallo, B.M.G., Silva, R.J. (2008b): Spirorchiids (Digenea: Spirorchiidae) infecting a Hawksbill sea turtle Eretmochelys imbricata (Linnaeus 1758) from Brazil. Arq. Bras. Med. Vet. Zootec., 60(3): $663-666$

Werneck, M.R., LIMA, E.H.S.M., PIRES, T., Silva, R.J. (2015b): Helminth parasites of the Juvenile Hawksbill Turtle Eretmochelys imbricata (Testudines, Cheloniidae) in Brazil. J. Parasitol., 101(4): 130 - 134. DOI: http://dx.doi.org/10.1645/13-479.1

WerneCK, M.R., MedeiRos, L.S. (2016). Report of the fourth specimen of Amphiorchis solus (Simha a Chattopadhyaya, 1970) 46 years after the original description. Helminthologia. Advance online publication. DOI 10.1515/helmin-2016-0020

WERnECK, M.R., SiLVA, R.J. (2013): Occurrence of Amphiorchis indicus Gupta \& Mehrotra, 1981 (Digenea, Spirorchiidae) infecting Green Turtle Chelonia mydas Linnaeus, 1758 (Testudines, Cheloniidae) in Brazil. Braz. J. Biol., 73(1): 225 - 227

WERnECK, M.R., Silva, R.J. (2015): Some helminth parasites of juvenile green turtles Chelonia mydas (Testudines, Cheloniidae) in Brazil. J. Parasitol., 101(6): 713 - 716. DOI: 10.1645/15-780

WERnECK, M.R., SILVA, R.J. (2012): Styphlotrema solitaria Looss, 1899 (Digenea, Styphlotrematidae) infecting Eretmochelys imbricata (Linnaeus 1758) (Testudines, Chelonidae) in Brazil. Neotrop. Helminthol., 6(1): $121-125$

Werneck, M.R., SouzA, G., Berguer B.C. (2016b): Neospirorchis schistosomatoides Price1934 (Digenea: Spirorchiidae) infecting a Green Turtle, Chelonia mydas Linnaeus, 1758 (Testudines, Cheloniidae), from Brazil. Helminthologia, 53(1): 94 - 98. DOI: 10.1515/ helmin-2015-0074

Werneck, M.R., Souza, G., Berger, B.C., Trazzi, A., Ribeiro, R., Silva, M.A., Leandro, H.J., Carvalho, E.C.Q. (2015d): Pathologi- 
cal changes by Hapalotrema postorchis Rao 1976 (Digenea: Spirorchiidae) in a green turtle Chelonia mydas Linnaeus 1758 (Testudines, Cheloniidae) from Brazil. Helminthologia, 52(2): 148 - 154. DOI: 10.1515/helmin-2015-0027

Werneck, M.R., Souza, V.R., Trazzi, A., Berger, B.C. (2015e): Monticellius indicum Mehra, 1939 (Digenea: Spirorchiidae) in a Hawksbill Turtle, Eretmochelys imbricata Linnaeus 1766 (Testudines, Cheloniidae) from Brazil. Comp. Parasitol., 82(1): 155 - 157. DOI: 10.1654/4716.1

Werneck, M.R., Thomazinı C.M., Mori, E.S., Gonçalves, V.T. GalLO, B.M., SILVA, R.J. (2008a): Gastrointestinal helminth parasites of Loggerhead turtle Caretta caretta Linnaeus 1758 (Testudines, Cheloniidae) in Brazil. PanamJAS, 3(3): 351 - 354

Werneck, M.R., Verissimo, L., Baldassin, P., Gagliardi, F., Tadashi, E., Vanderlinde, J. Baptistotte, C., Melo M.T.D., Lima, E.H.S.M., Gallo, B.M. G., Silva, R.J. (2012): Digenetic Trematodes of Dermochelys coriacea From the Southwestern Atlantic Ocean. Marine Turtles Newsletter, 132: 13 - 14

WoRMS- World Register of Marine Species (2015): Retrieved November 25, from: http://www.marinespecies.org/aphia.php?p=search 